\title{
Nodal tumor response according to the count of peripheral blood lymphocyte subpopulations during preoperative chemoradiotherapy in locally advanced rectal cancer
}

\author{
Jaesung Heo, MD, MS', Young-Taek Oh, MD, PhD ${ }^{1 *}, 0$ Kyu Noh, MD, PhD ${ }^{1 *}$, Mison Chun, MD, PhD, \\ Jun-Eun Park, MD, PhD², Sung-Ran Cho, MD, PhD ${ }^{3}$ \\ Departments of ${ }^{1}$ Radiation Oncology, ${ }^{2}$ Pediatrics, and ${ }^{3}$ Laboratory Medicine, Ajou University School of Medicine, Suwon, Korea
}

Purpose: The objective of this prospective study was to evaluate the relationship between the circulating lymphocyte subpopulation counts during preoperative chemoradiotherapy (CRT) and tumor response in locally advanced rectal cancer.

Materials and Methods: From August 2015 to June 2016, 10 patients treated with preoperative CRT followed by surgery were enrolled. Patients received conventional fractionated radiotherapy (50.4 Gy) with fluorouracil-based chemotherapy. Surgical resection was performed at 4 to 8 weeks after the completion of preoperative CRT. The absolute blood lymphocyte subpopulation was obtained prior to and after 4 weeks of CRT. We analyzed the association between a tumor response and change in the lymphocyte subpopulation during CRT.

Results: Among 10 patients, 2 (20\%) had evidence of pathologic complete response. In 8 patients with clinically node positive, 4 (50\%) had nodal tumor response. All lymphocyte subpopulation counts at 4 weeks after CRT were significantly lower than those observed during pretreatment $(p<0.01)$. A high decrease in natural killer (NK) cell, count during CRT (baseline cell count - cell count at 4 weeks) was associated with node down staging $(p=0.034)$.

Conclusion: Our results suggest that the change of lymphocyte subset to preoperative CRT may be a predictive factor for tumor response in rectal cancer.

Keywords: Rectal neoplasms, Immunity, Lymphocytes, NK cells

\section{Introduction}

In patients with locally advanced rectal cancer, preoperative chemoradiotherapy (CRT) is administered because of advantages such as better local control, a higher rate of sphincter preservation, and lower toxicity compared to postoperative CRT [1]. It also offers the chance of early tumor response evaluation that provides information regarding the degree of response to CRT and prognosis. Tumor response to preoperative CRT is associated with long-term prognosis [2].

Received 12 August 2016, Revised 9 September 2016, Accepted 12 September 2016.

Correspondence: Young-Taek Oh, MD, PhD, Department of Radiation Oncology, Ajou University School of Medicine, 206 World cup-ro, Yeongtong-gu, Suwon 16499, Korea. Tel: +82-31-219-5338, Fax: +82-31-219-5894, E-mail: ohyoung1963@ gmail.com

O Kyu Noh, MD, PhD, Department of Radiation Oncology, Ajou University School of Medicine, 206 World cup-ro, Yeongtong-gu, Suwon 16499, Korea. Tel: +82-31-219-5336, Fax: +82-31-219-5894, E-mail: okyu.noh@gmail.com

*Young-Taek Oh and $O$ Kyu Noh are contributed for this work equally.

(C) This is an Open Access article distributed under the terms of the Creative Commons Attribution Non-Commercial License (http://creativecommons.org/ licenses/by-nc/4.0/) which permits unrestricted non-commercial use, distribution, and reproduction in any medium, provided the original work is properly cited.

www.e-roj.org 
In previous studies, researchers attempted to identify the predictive factors that influence tumor response to CRT for rectal cancer. Clinical factors such as carcinoembryonic antigen (CEA), distance from the anal verge, and molecular profile were associated with tumor response $[3,4]$. Recently, in addition to the clinical factors, the relationship between host immunity and tumor response was studied. Circulating lymphocyte count in peripheral blood was found to be an important parameter to determine clinical outcomes in advanced rectal cancer [5]. We also recently confirmed that sustaining lymphocyte count during preoperative CRT could be a predictive factor in rectal cancer [6].

Lymphocytes are divided into several subtypes, and each type reflects host immunity. Subpopulations of lymphocytes before CRT in rectal cancer were analyzed, and the density of pretreatment CD8+ lymphocytes in tumor-infiltrating lymphocytes was found to be an independent predictive factor for tumor response [7]. In addition, subpopulations of lymphocytes during radiotherapy (RT) change and each subpopulation responds differently to RT [8]. In cancer patients, total lymphocyte count and $\mathrm{CD} 4^{+}$lymphocyte count decrease during RT; however, regulatory T-cell lymphocytes show no change at the end of pelvic irradiation [9]. These changes of lymphocyte subsets to treatment could influence host immunity and tumor response [10]. However, the correlation between these lymphocytic profile changes to treatment and tumor response has not been evaluated in advanced rectal cancer.

We analyzed the relationship between the circulating lymphocyte subpopulation level during preoperative CRT and tumor response in locally advanced rectal cancer.

\section{Materials and Methods}

\section{Patients}

In the prospective study, eligible patients had to satisfy all of the following criteria: 1) a histologically confirmed diagnosis of rectal cancer (adenocarcinoma); 2) resectable clinical stage T3-T4 or lymph-node metastasis positive based on computed tomography (CT) or magnetic resonance imaging (MRI); 3) CRT planned before surgery; 4) ECOG performance status of 0 or 1 ; 5) an age of $18-75$ years at enrollment; 6 ) no severe organ dysfunction; and 7) no history of other cancer within 5 years. Patients with distant metastasis, previous RT, or pregnancy were excluded. Before enrollment, written informed consent was obtained from all patients. Patients underwent preoperative staging work up, including complete blood count
(CBC) with differentiation, colonoscopy, MRI, and CT. Absolute lymphocyte counts and a subpopulation analysis were performed prior to and 4 weeks after the initiation of CRT. This study was reviewed and approved by the Institutional Review Board of Ajou University School of Medicine (AJIRB-MEDSMP-15-207).

\section{Treatments}

$\mathrm{RT}$ was delivered with 6- or 10-MV photon beams in $1.8 \mathrm{~Gy}$ daily to a total dose of $50.4 \mathrm{~Gy}$. Gross tumor volume (GTV) included the primary tumor and enlarged lymph nodes. The primary lesion was confirmed by MRI and CT. The clinical target volume (CTV) for the whole pelvis included the GTV, mesorectum, presacral area, and regional lymphatics (pararectal lymph nodes, internal iliac lymph nodes, and obturator lymph nodes). The planning target volume (PTV) was derived by expanding the CTV by a margin of $0.5-1.0 \mathrm{~cm}$. A bolus of 5 -fluorouracil ( $400 \mathrm{mg} / \mathrm{m}^{2} /$ day) and leucovorin $\left(20 \mathrm{mg} / \mathrm{m}^{2} /\right.$ day) were concurrently administered for 5 days in the first and fifth week of RT. In the ninth week, the 3rd chemotherapy could be administered depending on physician's decision based on the tumor response using clinical imaging and physical examination. Surgery was performed 4-8 weeks after the completion of CRT. Patients underwent low anterior resection or abdominoperineal resection (APR).

\section{Flow cytometry}

Blood was collected two times before and 4 weeks after the initiation of CRT. Lymphocyte subpopulations (CD3 ${ }^{+} \mathrm{T}$ lymphocyte, $\mathrm{CD}^{+} / \mathrm{CD}^{+}$helper $\mathrm{T}$ Iymphocytes, $\mathrm{CD} 3^{+} / \mathrm{CD} 8^{+}$ cytotoxic T lymphocyte, $\mathrm{CD}^{-} / \mathrm{CD} 19^{+} \mathrm{B}$ lymphocytes, and $\mathrm{CD} 3^{-}$ $\mathrm{CD} 6^{+}$natural killer cell [NK cell]) counts (cells/ $\mu \mathrm{L}$ ) and $\mathrm{CD}^{+} /$ $\mathrm{CD}^{+}$ratio were analyzed by multicolor flow cytometry. Acquired cell were analyzed by flow cytometry using a FC-500 cytometer (Beckman Coulter Inc., Brea, CA, USA). Monoclonal antibodies were used for cell surface staining. Instrument setting parameters such as threshold were set with machine software (CXP Software; Beckman Coulter Inc.) in conjunction with calibration beads (Flow-Set beads, CYTO-COMP kit, and CYTO-TROL Control Cells). Data were analyzed with CXP analysis software.

\section{Statistics}

The endpoint of this study was to estimate tumor regression grade, pathologic complete response ( $p C R$ ), lymph node response and the curative resection (RO) rate. Baseline evaluations were performed through imaging studies based 
on diffusion-weighted MRI with conventional MRI and CT. We used the sustaining ratio (SR) as a method of estimating CRT sensitivity of the lymphocyte subset. The SR was calculated by dividing the 4 weeks count by the pretreatment count.

Achieving a pCR was defined as a complete absence of any tumor cells, in both the primary site and dissected lymph nodes in the surgical specimens. Tumor and nodal response was evaluated by comparing the pretreatment status (determined by clinical staging) to the pathologic status after surgery. Node down staging was evaluated only among patients

Table 1. Patients' characteristics $(n=10)$

\begin{tabular}{lc}
\hline \multicolumn{1}{c}{ Characteristic } & Value \\
\hline Sex & \\
Male & $6(60)$ \\
Female & $4(40)$ \\
Age $(\mathrm{yr})$ & $59(48-72)$ \\
Pre-CRT CEA level $(\mathrm{ng} / \mathrm{mL})$ & \\
$\leq 5$ & $6(60)$ \\
$>5$ & $4(40)$ \\
Histology type & \\
Adenocarcinoma & $10(100)$ \\
Clinical T stage & \\
cT2 & $0(0)$ \\
cT3 & $10(100)$ \\
Clinical N stage & \\
cN0 & $2(20)$ \\
cN1-2 & $8(80)$ \\
Chemotherapy regimen & \\
5 -Fluorouracil & $10(100)$ \\
Chemotherapy cycle & \\
2 & $6(60)$ \\
3 & $4(40)$ \\
\hline
\end{tabular}

Values are presented as number (\%) or median (range). CRT, chemoradiotherapy; CEA, carcinoembryonic antigen. with clinically node positive. Clinical lymph node staging was evaluated based on signal intensity, border contour and size using T2 weighted MRI [11]. Differences in changes of lymphocyte subset counts between pretreatment and 4 weeks after the initiating CRT were analyzed by the Wilcoxon signed rank test. To confirm the relationship between Iymphocyte subpopulation and tumor response, the Mann-Whitney $U$ test was used. We assessed pattern over time in lymphocyte subset count by comparing tumor response group and non-tumor response group before and 4 weeks after initiation of CRT. We used a Friedman test as a nonparametric interaction test for factorial designs with repeated measures using an $\mathrm{R}$ program 'nplntFactRep' package [12]. Two-sided p-values less than 0.05 were considered to be significantly different. All statistical analyses were performed using $\mathrm{R}$ statistical packages [13].

\section{Results}

From August 2015 to June 2016, a total of 10 patients were enrolled. The clinical characteristics of the patients are shown in Table 1. Their ages ranged from 48 to 72 years, and 6 of the patients were men. All patients had clinical T3 staging and their node staging was No (2), N1-2 (8). Six patients received 2 cycles of chemotherapy and 4 patients received 3 cycles based on 5-fluorouracil. All 10 patients had lower anterior resection (100\%) because of the tumor location.

The tumor response of preoperative CRT was evaluated in all 10 patients (Table 2). The pCR rate was 20\%, and RO resection was 100\%. T down staging was confirmed in 4 patients (40\%). Node down staging which changed from clinical node positive to pathologic node negative after CCRT was occurred 4 of 8 patients with node positive. The median SR (mSR) for the lymphocyte was 0.31 and $\mathrm{mSR}$ for B lymphocytes was 0.11 ,

Table 2. Tumor response

\begin{tabular}{|c|c|c|c|c|c|c|}
\hline & Before treatment & After treatment & $\mathrm{pCR}$ & T down staging & $\mathrm{N}$ down staging & R0 resection \\
\hline 1 & T3N2M0 & ypT3N0M0 & No & No & Yes & Yes \\
\hline 2 & T3N2Mo & ypT3N1M0 & No & No & No & Yes \\
\hline 3 & T3N1M0 & yрT2NOMO & No & Yes & Yes & Yes \\
\hline 4 & T3N0MO & ypT2NOMO & No & Yes & - & Yes \\
\hline 5 & T3N2M0 & ypT3N1M0 & No & No & No & Yes \\
\hline 6 & T3N2M0 & ypT3N1M0 & No & No & No & Yes \\
\hline 7 & T3N1M0 & ypT3N1M0 & No & No & No & Yes \\
\hline 8 & T3N0MO & ypT3N2M0 & No & No & - & Yes \\
\hline 9 & T3N1M0 & ypTONOMO & Yes & Yes & Yes & Yes \\
\hline 10 & T3N2M0 & ypTONOMO & Yes & Yes & Yes & Yes \\
\hline
\end{tabular}

$\mathrm{pCR}$, pathologic complete response. 
Table 3. Clinical parameters of node down staging $(n=4)$ and non-node down staging to the preoperative CRT $(n=4)$

\begin{tabular}{|c|c|c|c|c|}
\hline & Variable & Node DS (+) & Node DS (-) & $p$-value \\
\hline \multirow[t]{14}{*}{ Baseline } & CEA (ng/mL) & $3.95(1.6-15.4)$ & $8.2(2.9-23.1)$ & 0.386 \\
\hline & WBC $\left(\times 10^{9} / \mathrm{L}\right)$ & $6,850(4,000-8,500)$ & $6,600(5,100-6,500)$ & 0.243 \\
\hline & Lymphocyte (×10 $/ \mathrm{L})$ & $1,850(1,700-2,800)$ & $1,850(1,400-2,200)$ & 0.561 \\
\hline & B lymphocyte (\%) & $15(10-23)$ & $13(10-14)$ & 0.661 \\
\hline & B lymphocyte (×109/L) & $330(180-500)$ & $250(140-290)$ & 0.564 \\
\hline & T Iymphocyte (\%) & $69(54-79)$ & $73(69-78)$ & 0.554 \\
\hline & T lymphocyte (×109/L) & $1,255(1,100-1,780)$ & $1,385(960-1,630)$ & 0.885 \\
\hline & Th $(\%)$ & $42(36-52)$ & $48(39-56)$ & 0.386 \\
\hline & $\operatorname{Th}\left(\times 10^{9} / \mathrm{L}\right)$ & $835(720-1,020)$ & $800(780-1,060)$ & 1.000 \\
\hline & CTL $(\%)$ & $24(14-33)$ & $24(12-37)$ & 1.000 \\
\hline & $\mathrm{CTL}\left(\times 10^{9} / \mathrm{L}\right)$ & $450(280-720)$ & $440(170-790)$ & 1.000 \\
\hline & CD4/CD8 ratio & $1.95(1.28-2.90)$ & $2.12(1.05-4.69)$ & 0.773 \\
\hline & NK cell (\%) & $15(10-16)$ & $13(6-20)$ & 0.564 \\
\hline & NK cell $\left(\times 10^{9} / \mathrm{L}\right)$ & $280(170-420)$ & $235(120-280)$ & 0.384 \\
\hline \multirow[t]{20}{*}{ At 4 weeks } & CEA (ng/mL) & $1.6(1.3-10.7)$ & $6.5(2.8-25.8)$ & 0.154 \\
\hline & WBC $\left(\times 10^{9} / \mathrm{L}\right)$ & $4,650(3,300-5,100)$ & $4,400(4,300-7,500)$ & 0.476 \\
\hline & Lymphocyte (×10 $/ \mathrm{L})$ & $600(500-800)$ & $900(500-1,100)$ & 0.271 \\
\hline & B lymphocyte (\%) & $4(3-7)$ & $4(3-8)$ & 0.853 \\
\hline & B lymphocyte (×109/L) & $25(20-50)$ & $30(20-70)$ & 0.578 \\
\hline & T lymphocyte (\%) & $68(67-77)$ & $71(56-85)$ & 0.721 \\
\hline & T lymphocyte $\left(\times 10^{9} / \mathrm{L}\right)$ & $440(330-570)$ & $780(300-800)$ & 0.480 \\
\hline & Th $(\%)$ & $41(39-53)$ & $47(35-48)$ & 1.000 \\
\hline & $\operatorname{Th}\left(\times 10^{9} / \mathrm{L}\right)$ & $260(200-380)$ & $440(190-530)$ & 0.480 \\
\hline & CTL (\%) & $27(14-36)$ & $25(19-36)$ & 0.858 \\
\hline & $\mathrm{CTL}\left(\times 10^{9} / \mathrm{L}\right)$ & $155(100-230)$ & $220(130-330)$ & 0.372 \\
\hline & $\mathrm{CD}^{+} / \mathrm{CD}^{+}$ratio & 1.51 (1.09-3.73) & $1.40(1.33-2.4)$ & 1.000 \\
\hline & NK cell (\%) & $23(19-28)$ & $26(7-32)$ & 0.857 \\
\hline & NK cell $\left(\times 10^{9} / \mathrm{L}\right)$ & $140(100-290)$ & $180(60-290)$ & 0.721 \\
\hline & Decreased lymphocyte & $1,250(200-2,000)$ & $1,100(1,100-1,200)$ & 0.064 \\
\hline & Decreased B lymphocyte & $296(160-470)$ & $220(200-250)$ & 1.000 \\
\hline & Decreased T Iymphocyte & $890(620-1210)$ & 850 (770-900) & 0.480 \\
\hline & Decreased Th & $580(430-710)$ & $530(380-590)$ & 0.480 \\
\hline & Decreased CTL & 295 (180-490) & $340(190-460)$ & 1.000 \\
\hline & Decreased NK cell & $140(70-210)$ & $10(10-60)$ & $0.034^{*}$ \\
\hline
\end{tabular}

Values are presented as median (range).

DS, down staging; CEA, carcinoembryonic antigen; WBC, white blood cell; Th, helper T lymphocyte; CTL, cytotoxic T Iymphocyte; NK cell, natural killer cell.

${ }^{*} p<0.05$.

which was the lowest compared to the other subpopulation values (Fig. 1). The mSR for NK cells was 0.55 .

The lymphocyte subset counts according to node down staging between pretreatment and 4 weeks after treatment are shown in Fig. 1. At the pretreatment time point and 4 weeks after the initiation of treatment, there was no difference in clinical parameters between the node downs staging group versus the non-node down staging group to CRT (Table 3 ). However, all lymphocyte subpopulation counts at 4 weeks after the start of treatment were significantly decreased compared to the baseline result ( $p<0.05$ ).

We evaluated the relationship between Iymphocyte subpopulations and tumor response to preoperative CRT. The factors in relationship to a pCR were not confirmed; however, the $\mathrm{CD} 4^{+} / \mathrm{CD}^{+}$ratio at 4 weeks after the initiation of CRT exhibited a trend ( $p=0.079$ ). T down staging was not associated with clinical parameters. Among patients with lymph node positive, decreased Iymphocyte count had a tendency to have an association with nodal response ( $p=$ 0.067). In analysis of lymphocyte subset, a pattern of NK cell 
ALC

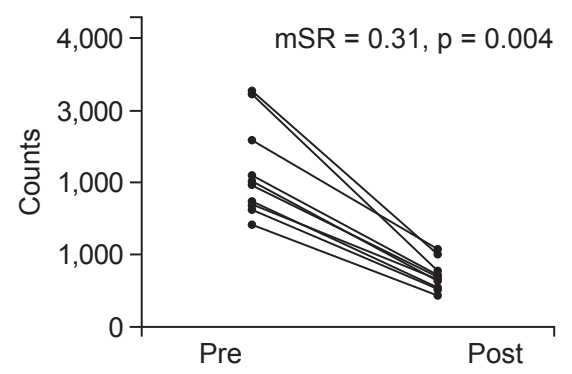

T lymphocyte

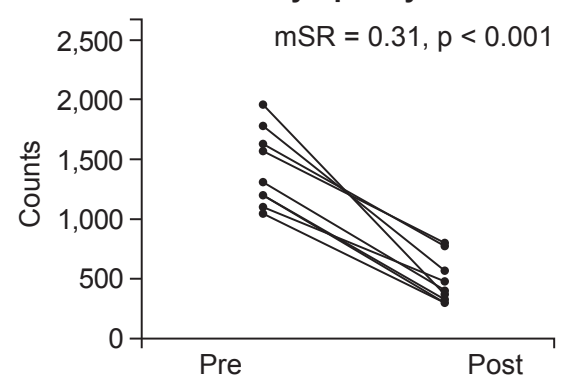

B lymphocyte

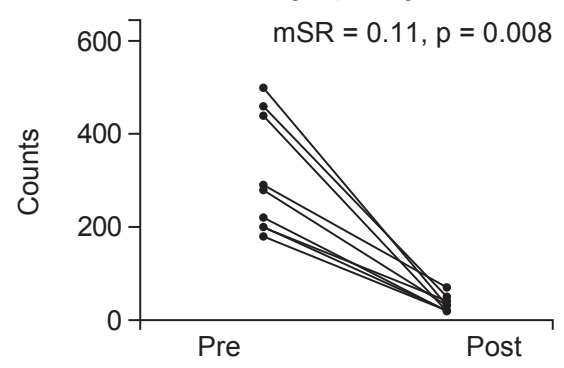

Th

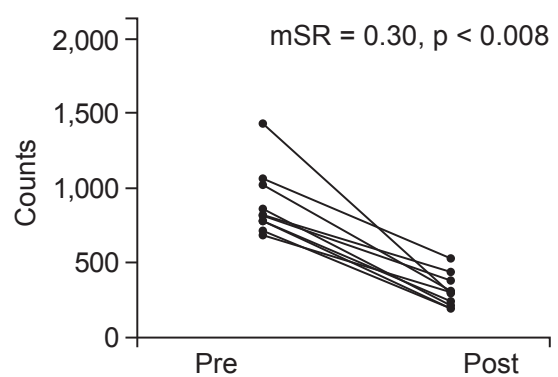

NK cell

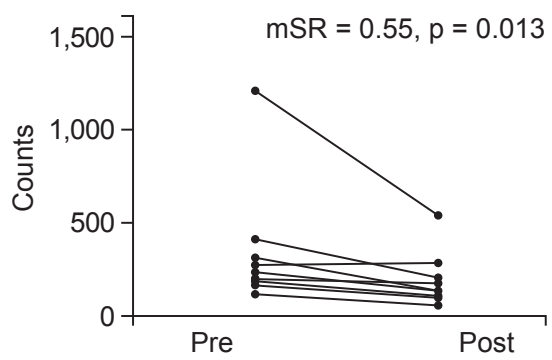

CTL

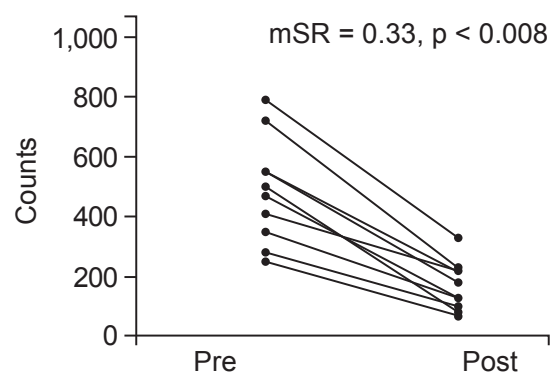

Fig. 1. Changes in the lymphocyte subpopulations between baseline and 4 weeks after the start of treatment. ALC, absolute lymphocyte count; NK cell, natural killer cell; Th, helper T lymphocyte; CTL, cytotoxic T lymphocyte; mSR, median sustaining ratio.

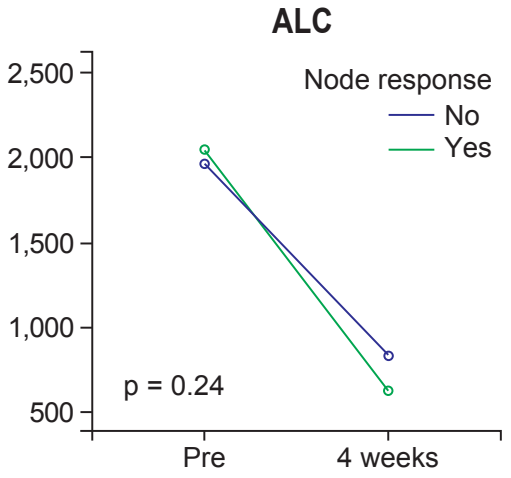

T lymphocyte

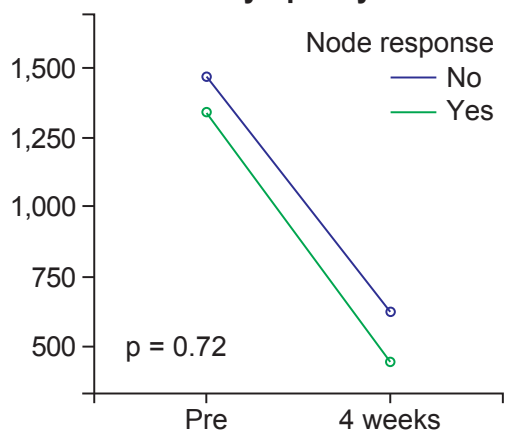

NK cell

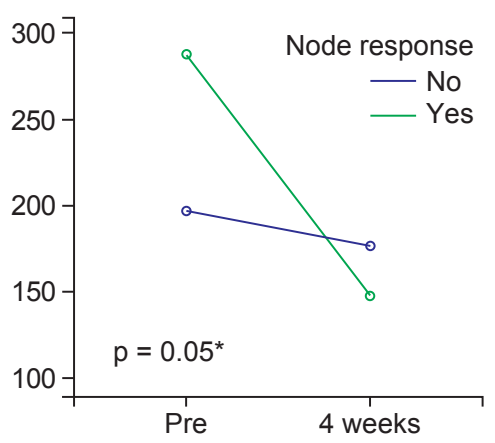

Th

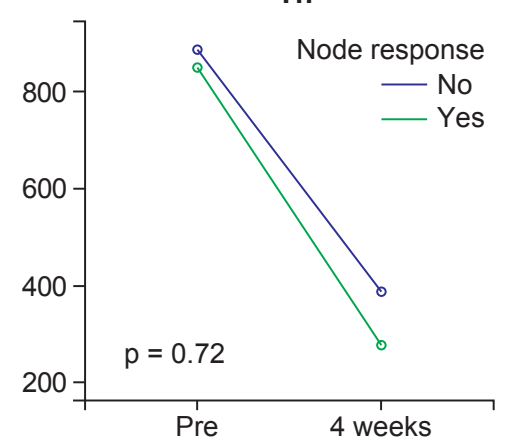

B cell

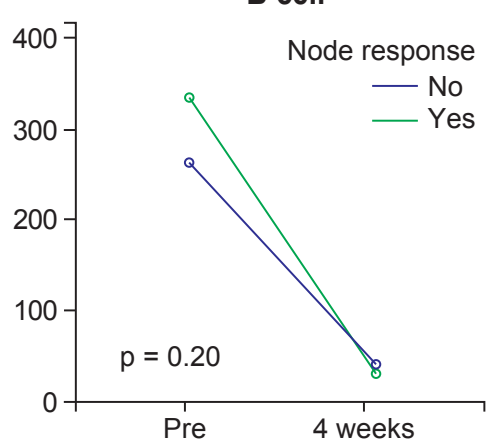

CTL

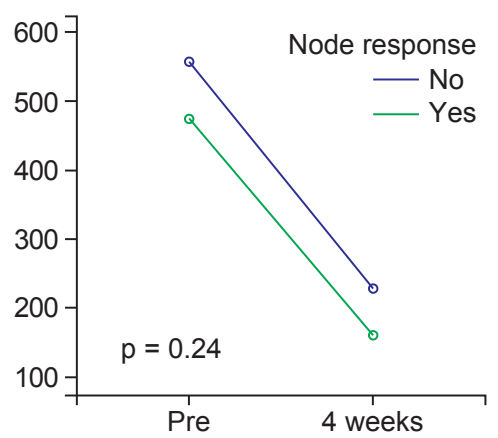

Fig. 2. Pattern of changes in the lymphocyte subpopulations during chemoradiotherapy according to $\mathrm{N}$ down staging. ALC, absolute Iymphocyte count; NK cell, natural killer cell; Th, helper T Iymphocyte; CTL, cytotoxic T Iymphocyte. * $p<0.05$. 
change between before and after CRT showed a trend for the association with nodal response ( $p=0.052$ by repeated measure analysis) (Fig. 2). Also decreased NK cell count at 4-week (NK cell count at baseline - NK cell count at 4 weeks after initiating treatment) was associated with nodal response ( $p=0.034$ ) (Table 3 and Fig. 3).

\section{Discussion and Conclusion}

Lymphocyte subtypes include T Iymphocytes, B lymphocytes, and natural killer cells. Each subpopulation of Iymphocytes has different immune functions and different responses to CRT [14]. In a previous study, we showed that change in lymphocytes during CRT was associated with a pCR in locally advanced rectal cancer [6]. This result inspired us to evaluate the relationship between subpopulations of lymphocytes and tumor response. In this study, we found that change of NK cell showed a trend of correlation with nodal tumor response.

Tumor response to preoperative CRT is dependent on a patient's status. We could select treatment plan or modality based on predictions of response. Many studies focused on clinical factors and molecular profile of cancer lesion and confirmed the association of biomarker with tumor response $[3,4]$. Immunological host status also influenced tumor response [5]. Previous studies also considered pretreatment immune status such as the density of CD4(+) and CD8(+) T lymphocyte [15]. However, during preoperative CRT, host immunity status was changed. We confirmed that the lymphocyte subset profile before and 4 weeks after initiation of CRT was significantly decreased (Fig. 1).

Therefore, change of host immune status could influence tumor response. Decreased peripheral blood Iymphocytes count based on radiation-induced apoptosis was associated with histological tumor regression to preoperative CRT in rectal cancer [16]. High proportion of apoptosis in lymphocyte showed good histological regression to treatment. In our study, decreased lymphocyte count also showed a trend in association with node down staging ( $p=0.067)$ (Fig. 3). In the study using lymphocyte subset analysis, higher reduction of $T$ lymphocyte containing Th and Tc during CRT was associated with T down staging [17].

In addition to $T$ down staging, we found that change of NK cell during treatment was related to lymph node down staging. The pattern and amount of change in NK cells during CRT were associated with pathologic lymph node metastasis (Figs. 2 and 3). These results suggested that the change of lymphocyte and lymphocyte subset in peripheral blood could be used as a biomarker to predict tumor response. Especially, in patients with rectal cancer, pathologic lymph node status was associated with prognosis [18]. Therefore, prediction of lymph node response using change to CRT could be useful to decide treatment plan after CRT. If poor node response to CRT is predicted, we could consider surgery rather than additional chemotherapy after CRT. Besides N down staging, pCR and T down staging were not associated with change of NK cell (Fig. 4).

In this study, we used the SR as a method of estimating sensitivity of the lymphocyte subset to treatment. The Iymphocyte subpopulation also has a different response to chemotherapy and radiotherapy $[8,19]$. Sensitivity to CRT for

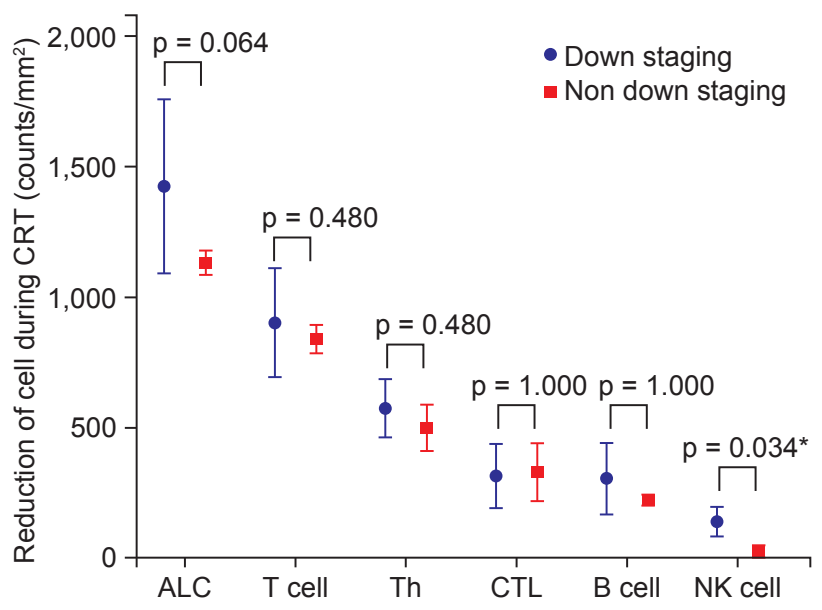

Fig. 3. The correlation between the decreased in numbers of lymphocyte subset during chemoradiotherapy (CRT) and $\mathrm{N}$ down staging. ALC, absolute lymphocyte count; Th, helper T lymphocyte; CTL, cytotoxic T lymphocyte; NK cell, natural killer cell. ${ }^{*} p<0.05$.

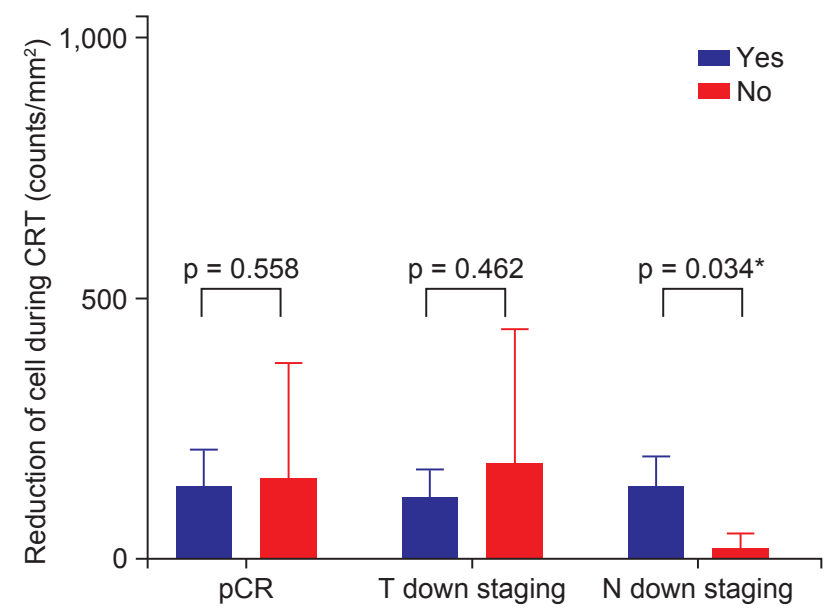

Fig. 4. The correlation between tumor response and reduction in numbers of NK cells. CRT, chemoradiotherapy; $p C R$, pathologic complete response. ${ }^{*} p<0.05$. 
NK cells was the lowest in the lymphocyte subset $(\mathrm{mSR}=$ 0.55). The $\mathrm{mSR}$ for NK cells was 5 times higher than that for $B$ cells which was most sensitive to CRT (0.55 vs. 0.11). The mSR for cytotoxic T lymphocyte (CTL) was 0.33 and CTL showed more resistance compared to Th (mSR 0.33 vs. 0.30) (Fig. 1). Since NK cell is most resistant to CRT, change of NK cell could more critical to tumor response than any another lymphocyte subset. In fact, mSR for NK cell in according to node down staging was different (0.54 vs. 0.95) and node down staging group had low mSR.

Besides divergent sensitivity to CRT, Iymphocyte subpopulation has a different immune function. NK cells are cytotoxic lymphocytes that are part of the innate immune system [20]. In tumors, NK cells eliminate tumor cells without activation of antigens. CTL has the function of adaptive immune responses based on cell mediated immunity [21]. CTL recognizes tumor antigens by Th and kills tumor cells. Th has a function of recognizing antigens on the surface of antigen presenting cells (APC) and secrete cytokines for differentiation from naïve CTL cells into activated CTL [22]. We confirmed that a low $\mathrm{CD} 4^{+} / \mathrm{CD}^{+}$ratio has a tendency to have a relationship with $\mathrm{pCR}(\mathrm{p}=0.079)$. The $\mathrm{CD} 4^{+} / \mathrm{CD} 8^{+}$ratio indicates immune status and has clinical significant relation with lymph node metastasis in cervical cancer [23].

$B$ Iymphocytes originate from the bone marrow and are related to humoral immunity. B Iymphocytes have been reported to be the most radiation sensitive subpopulation type [8]. In breast cancer patients, circulating B lymphocytes during fractionated RT show severe depletion. After completion of RT, a gradual recovery has been confirmed [14]. In addition, B cells are sensitive to chemotherapy [19]. The mSR for B lymphocytes was 0.11 and it was the lowest of the subpopulation types. Absolute counts of B lymphocytes decreased significantly after CRT (Fig. 1). However, in the analysis of their relationship with tumor response, B Iymphocytes were not significantly associated with $\mathrm{pCR}$, T and $\mathrm{N}$ down staging.

There are some limitations in this study. Unlike previous studies using tumor infiltrating lymphocyte (TIL), we analyzed circulating lymphocyte subset. The correlation between circulating lymphocyte and TIL has not been confirmed. However, naive lymphocytes continuously migrate from the blood into the lymph nodes. Activated lymphocytes in lymph nodes move to specific sites through peripheral blood vessels. The pelvic area receiving $\mathrm{RT}$ has regional Iymphatics and vessels near lymph nodes. Therefore, evaluation of circulating lymphocytes in rectal cancer could reflect patient immunity. High peripheral lymphocytes have association with favorable prognosis [15]. Specimen to evaluate TIL after surgery was affected by CRT and could not reflect primary status. Also, biopsy specimen before CRT could not represent whole cancer tissue. Although prospective study was conducted, sample size was relatively small. Relation between lymphocyte subpopulation and tumor response was analyzed with nonparametric method such as Wilcoxon signed rank test and the Mann-Whitney U test. Also, to avoid variability due to diurnal changes, blood sampling was twice implemented before and 4 weeks after initiating CRT.

In conclusion, Host immunity plays an important role in tumor responses to CRT for rectal cancer. Each Iymphocyte subpopulation responded differently to CRT and had different functions. B lymphocytes were the most sensitive while NK cells were the most resistant to CRT. NK cell change during CRT were associated with nodal tumor response. Further studies on large patient populations are warranted to confirm our observations.

\section{Conflict of Interest}

No potential conflict of interest relevant to this article was reported.

\section{Acknowledgments}

This work was supported by the new faculty research fund of Ajou University School of Medicine. The authors thank Stephen Kim for assistance with manuscript editing.

\section{References}

1. Sauer $R$, Becker $H_{1}$ Hohenberger $W$, et al. Preoperative versus postoperative chemoradiotherapy for rectal cancer. N Engl J Med 2004;351:1731-40.

2. Walker AS, Zwintscher NP, Johnson EK, et al. Future directions for monitoring treatment response in colorectal cancer. $J$ Cancer 2014:5:44-57.

3. Restivo A, Zorcolo L, Cocco IM, et al. Elevated CEA levels and low distance of the tumor from the anal verge are predictors of incomplete response to chemoradiation in patients with rectal cancer. Ann Surg Oncol 2013;20:864-71.

4. Kuremsky JG, Tepper JE, McLeod HL. Biomarkers for response to neoadjuvant chemoradiation for rectal cancer. Int J Radiat Oncol Biol Phys 2009;74:673-88.

5. Kitayama J, Yasuda K, Kawai K, Sunami E, Nagawa H. Circulating lymphocyte is an important determinant of the effectiveness of preoperative radiotherapy in advanced rectal 
cancer. BMC Cancer 2011;11:64.

6. Heo J, Chun M, Noh OK, et al. Sustaining blood lymphocyte count during preoperative chemoradiotherapy as a predictive marker for pathologic complete response in locally advanced rectal cancer. Cancer Res Treat 2016;48:232-9.

7. Yasuda K, Nirei T, Sunami E, Nagawa H, Kitayama J. Density of CD4(+) and CD8(+) T lymphocytes in biopsy samples can be a predictor of pathological response to chemoradiotherapy (CRT) for rectal cancer. Radiat Oncol 2011;6:49.

8. Heylmann D, Rodel F, Kindler T, Kaina B. Radiation sensitivity of human and murine peripheral blood lymphocytes, stem and progenitor cells. Biochim Biophys Acta 2014;1846:121-9.

9. Lissoni P, Brivio F, Fumagalli $L$, et al. Effects of the conventional antitumor therapies surgery, chemotherapy, radiotherapy and immunotherapy on regulatory $T$ lymphocytes in cancer patients. Anticancer Res 2009;29:1847-52.

10. Garcia-Martinez E, Gil GL, Benito AC, et al. Tumor-infiltrating immune cell profiles and their change after neoadjuvant chemotherapy predict response and prognosis of breast cancer. Breast Cancer Res 2014;16:488.

11. Beets-Tan RG, Lambregts DM, Maas $M$, et al. Magnetic resonance imaging for the clinical management of rectal cancer patients: recommendations from the 2012 European Society of Gastrointestinal and Abdominal Radiology (ESGAR) consensus meeting. Eur Radiol 2013;23:2522-31

12. Feys J. npIntFactRep: nonparametric interaction tests for factorial designs with repeated measures [Internet]. Vienna, Austria: The R Foundation for Statistical Computing; c2015 [cited 2016 Oct 20]. Available from: https://cran.r-project.org/ web/packages/nplntFactRep/nplntFactRep.pdf.

13. R Core Team. R: a language and environment for the statistical computing [Internet]. Vienna, Austria; The R Foundation for Statistical Computing; c2012 [cited 2016 Oct 20]. Available from: https://cran.r-project.org/doc/manuals/r-release/ fullrefman.pdf.

14. Sage EK, Schmid TE, Sedelmayr M, et al. Comparative analysis of the effects of radiotherapy versus radiotherapy after adjuvant chemotherapy on the composition of Iymphocyte subpopulations in breast cancer patients. Radiother Oncol 2016;118:176-80.

15. He JR, Shen GP, Ren ZF, et al. Pretreatment levels of peripheral neutrophils and lymphocytes as independent prognostic factors in patients with nasopharyngeal carcinoma. Head Neck 2012;34:1769-76.

16. Ishihara $S$, linuma $H$, Fukushima $Y$, et al. Radiation-induced apoptosis of peripheral blood lymphocytes is correlated with histological regression of rectal cancer in response to preoperative chemoradiotherapy. Ann Surg Oncol 2012;19:1192-8.

17. Tada N, Kawai K, Tsuno NH, et al. Prediction of the preoperative chemoradiotherapy response for rectal cancer by peripheral blood lymphocyte subsets. World J Surg Oncol 2015;13:30.

18. Ong ML, Schofield JB. Assessment of lymph node involvement in colorectal cancer. World J Gastrointest Surg 2016;8:179-92.

19. Sewell HF, Halbert CF, Robins RA, Galvin A, Chan S, Blamey RW. Chemotherapy-induced differential changes in lymphocyte subsets and natural-killer-cell function in patients with advanced breast cancer. Int J Cancer 1993;55:735-8.

20. Marcus A, Gowen BG, Thompson TW, et al. Recognition of tumors by the innate immune system and natural killer cells. Adv Immunol. 2014;122:91-128.

21. Spurrell EL, Lockley M. Adaptive immunity in cancer immunology and therapeutics. Ecancermedicalscience 2014;8:441.

22. Knutson KL, Disis ML. Tumor antigen-specific $T$ helper cells in cancer immunity and immunotherapy. Cancer Immunol Immunother 2005;54:721-8.

23. Hadrup S, Donia M, thor Straten P. Effector CD4 and CD8 T Cells and Their Role in the Tumor Microenvironment. Cancer Microenviron 2013;6:123-33. 